\title{
Archéopages
}

Archéopages

Archéologie et société

34 | 07/2012

Campagnes

\section{Les figures du paysan et de la paysanne. Reliefs du pavillon soviétique à l'Exposition internationale de} 1937

Marie Vacher

\section{CpenEdition}

Journals

Édition électronique

URL : https://journals.openedition.org/archeopages/404

DOI : 10.4000/archeopages.404

ISSN : 2269-9872

Éditeur

INRAP - Institut national de recherches archéologiques préventives

Édition imprimée

Date de publication : 1 février 2012

Pagination : 74-77

ISSN : 1622-8545

\section{Référence électronique}

Marie Vacher, "Les figures du paysan et de la paysanne. Reliefs du pavillon soviétique à l'Exposition internationale de 1937 », Archéopages [En ligne], 34 | 07/2012, mis en ligne le 01 juillet 2012, consulté le 22 janvier 2022. URL : http://journals.openedition.org/archeopages/404 ; DOI : https://doi.org/ 10.4000/archeopages.404 


\title{
Les figures du paysan et de la paysanne Reliefs du pavillon soviétique à l'Exposition internationale de 1937
}

\author{
Marie Vacher Doctorantea ll'École du Louvre età lIINALCO
}

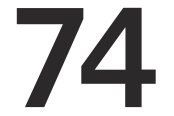

1. Ukraine, Biélorussie, Azerbaïdjan, Géorgie,

Arménie, Turkménistan, Ouzbékistan, Tadjikistan, Kazakhstan, Kirghizstan. 2. Ouvriers, ouvrières : le couple russe, l'homme ukrainien, l'homme azéri, l'homme arménien, l'homme kazakh. Paysans, paysannes : l'Ukrainienne, le couple biélorusse, la femme azérie, le couple géorgien, l'Arménienne, le couple turkmène, le couple ouzbek, le couple tadjik, la Kazakh, le couple kirghize. Pour plus de détails, voir

l'identification des

différentes allégories proposée par François Gentili.

3. « Le Monde paysan dans l'art russe », 2005
En 2004, un diagnostic mené par François Gentili à Baillet-en-France (Val-d'Oise), suivi d'une fouille en 2009, a permis la redécouverte des reliefs ornant les propylées du pavillon de l'URSS de l'exposition internationale de 1937 à Paris. Euvre de l'artiste russe Joseph Moiseevitch Tchaikov (1888-1979), ces sculptures monumentales en béton métallisé avaient été offertes par l'URSS à la CGT après le démontage du pavillon. Installés dans le parc de loisirs du château de Baillet-en-France, propriété de la CGT-Métallurgie, ces reliefs, brisés et jetés dans l'une des glacières du château, murée en 1954, sont tombés dans l'oubli (Dufils, Gentili, Vacher, 2011 ; Gentili, 2011 ; Gentili, Vacher, à paraître; Vaudour, 2011). Cette œuvre nous donne l'occasion de porter un regard nouveau sur le réalisme socialiste et les héros du panthéon soviétique. Valorisant les ressources naturelles d'un territoire en recomposition, ces reliefs mettent tout particulièrement en avant les figures du paysan et de la paysanne. Véritables allégories du territoire et symboles de l'abondance apportée par la collectivisation de l'agriculture, ils trouvent dans les années 1930 une place nouvelle dans l'iconographie soviétique.

\section{Le contexte de la commande : un affrontement idéologique}

Dans un affrontement architectural resté célèbre, le pavillon soviétique, construit par Boris Iofan sur l'esplanade du Trocadéro, se dressait face au pavillon allemand d'Albert Speer. Au sommet d'une tour haute de $33 \mathrm{~m}$, L'Ouvrier et la Kolkhozienne de Véra Moukhina brandissaient faucille et marteau. En contrebas, deux massifs latéraux accueillaient les visiteurs. Ces propylées à l'antique - longs de $20 \mathrm{~m}$ et hauts de $4 \mathrm{~m}$ - étaient ornés de sculptures colossales représentant les allégories des onze républiques soviétiques nouvellement définies dans la constitution du 15 décembre 1936.

Commandés à Tchaikov pour le vingtième anniversaire de la révolution d'Octobre, ces reliefs ont été imaginés, dès leur conception, comme un véritable manifeste du réalisme socialiste et de la constitution de 1936. Les sculptures devaient glorifier les différents peuples de l'URSS, unis au sein d'un même monument. Les deux massifs présentaient chacun, dans l'ordre donné par la nouvelle constitution, la république soviétique fédérative socialiste de Russie (RSFSR) et les dix républiques socialistes soviétiques (RSS) ${ }^{1}$. Deux panneaux venaient compléter l'ensemble aux extrémités de chaque massif : La Musique et la Danse, et Le Sport et l'Armée. Chaque république était représentée par son armoirie officielle et incarnée par un couple en costume traditionnel, sculpté dans des poses dévoilant les principales ressources économiques de chaque république. De façon surprenante en regard de la prédominance de la figure de l'ouvrier dans l'iconographie soviétique, les paysans et paysannes tiennent une place de choix dans le programme. Sur vingtdeux personnages, seize sont des figures représentées dans des activités de type agricole ${ }^{2}$ : production laitière, pêche, exploitation du bois, culture du blé ou du coton [ill. 1] (Gentili, 2010).

\section{La figure du paysan dans l'iconographie russe et soviétique \\ Une exposition du musée Russe de Saint-} Pétersbourg a récemment mis en valeur l'importance des figures du monde paysan dans la peinture russe, en particulier au XIX ${ }^{\mathrm{e}}$ siècle ${ }^{3}$. En représentant bien souvent ses fêtes et ses rites, l'artiste se faisait à l'époque le chroniqueur d'un monde aux valeurs en apparence immuables. Un monde dont la misère fut 
aussi montrée et dénoncée à partir des années 1870, par les peintres de la Société des Ambulants, à travers un réalisme qualifié de « critique ». Au début $\mathrm{du} \mathrm{XX}^{\mathrm{e}}$ siècle, ce sont les peintres néo-primitivistes, tels Mikhaill Larionov et Natalia Gontcharova, qui s'emparent à leur tour de scènes de la vie rurale, comme les moissons ou le blanchissement de la toile au soleil ${ }^{\mathbf{4}}$. La vraie rupture intervient avec la révolution d'Octobre. Ce monde paysan, gardien de traditions ancestrales et peu favorable au nouveau gouvernement, est considéré par les Bolchéviques avec suspicion. Bien que le Parti reconnaisse la nécessité d'une alliance avec la population rurale largement majoritaire en 1917 - et se hâte de proclamer la « dictature du prolétariat et de la paysannerie pauvre », les représentations des paysans vont être dans un premier temps écartées au profit de celles des ouvriers.

La rhétorique du Parti mettant l'accent sur la révolution prolétarienne et la dictature du prolétariat, la figure de l'ouvrier va dominer dans la propagande visuelle entre 1919 et 1930. Cependant, avec le lancement du premier plan quinquennal (1928-1932) et de la collectivisation de l'agriculture (1929-1932), la légitimation des réformes staliniennes va devenir une priorité. L'hure n'est plus à la sacralisation du prolétariat : la révolution, pour Staline, ne doit pas être faite par une seule classe mais par toute une société, par l'alliance de l'ouvrier et du paysan ; un paysan rapidement remplacé par la figure de la kolkhozienne qui devient l'héroïne du panthéon soviétique.

Si la paysanne entre dans l'iconographie soviétique dès les années 1920, il s'agit alors plutôt d'une robuste $b a b a$ incarnant de façon négative un monde paysan brutal et patriarcal. Mais, au tournant des années 1930, en réponse au rôle important des femmes dans les révoltes contre la collectivisation des terres, les artistes renversent cette iconographie. Ils créent l'image de la jeune kolkhozienne, farouche et énergique, menant les paysans. Brandissant sa fourche contre les koulaks et les prêtres ou conduisant un tracteur, la paysanne est la figure majeure de la propagande visuelle justifiant la politique agraire.

\section{Les reliefs de Tchaikov: une déclinaison de L'Ouvrier et la Kolkhozienne de Véra Moukhina}

Une projection de zinc en fusion, ou shoopage donnait au béton des reliefs un aspect métallisé Moukhina. Le jeu des matériaux permettait ainsi d'établir un lien direct entre les reliefs et l'Ouvrier et la Kolkhozienne [ill. 2].

L'œuvre de Véra Moukhina pour l'exposition internationale de 1937 est devenue une icône de l'URSS dans la période stalinienne. S'inspirant du groupe antique des Tyrannoctones ${ }^{\mathbf{5}}$, l'artiste en a repris les gestes et le dynamisme. Brandissant le marteau et la faucille, l'ouvrier et la kolkhozienne répondent aux nouveaux impératifs du réalisme socialiste : plus jeunes, plus expressifs, ils ne sont qui faisait écho à la tôle d'acier des statues de Véra plus seulement les symboles d'une classe mais fonctionnent comme un modèle pour toute une société. Engagés dans l'action, l'ouvrier et la kolkhozienne construisent le socialisme, en prototypes parfaits du nouvel homme et de la nouvelle femme soviétiques. L'action est en effet désormais primordiale dans les représentations, et domine également dans les reliefs de Tchaikov.

Reprenant la logique du couple, Tchaikov la décline pour chaque république. Les couples d'ouvriers alternent avec les couples de paysans ou les couples « mixtes » paysan-ouvrier dans une logique de juxtaposition révélatrice de la hiérarchie des héros soviétiques. C'est généralement la présence de l'ouvrier à ses côtés qui permet d'héroïser le paysan par un effet d'association visuelle (Bonnell, 1997). En l'absence d'ouvrier, la redondance du couple ou du groupe peut elle aussi permettre de faire accéder les paysans au rang de héros soviétiques. Il est à noter que les dominations de classe et de genre apparaissent inextricablement liées. Tout comme l'ouvrière, la kolkhozienne n'est que rarement représentée seule ; elle est presque toujours associée à un ouvrier ou un paysan, et ce, malgré l'importance qu'elle acquiert dans les années 1930. Les relations de contiguïté visuelle marquent ainsi la hiérarchie sociale malgré la volonté d'unir les différentes classes.

\section{La reconfiguration d'un monde social et politique}

Si les sculptures de Tchaikov sont profondément marquées par le contexte social et politique, elles ont aussi pour rôle de participer à la reconfiguration en cours du monde soviétique. Agissant comme des opérateurs, les images du réalisme socialiste fonctionnent comme un idéal type à atteindre ; le spectateur étant partie intégrante de l'œuvre. La question du tipazh devient alors essentielle. Dans le vocabulaire soviétique, il s'agit du rendu correct d'une catégorie sociale particulière. En d'autres termes, il est question de la représentation du présent mais également du futur en train d'advenir pour cette catégorie sociale. L'artiste doit donc représenter une réalité en développement.

Ce tipazh poursuivi par Tchaikov est des paysannes. Caractéristiques de la deuxième moitié des années 1930, elles sont moins agressives que la kolkhozienne athlétique des débuts de la collectivisation. Elles semblent plus calmes, plus sereines et plus mûres. Éléments caractéristiques du tipazh, leurs vêtements et leurs coiffes font échos aux récentes campagnes gouvernementales qui mettent en avant la propreté et les beaux vêtements. Les représentations intègrent alors des vêtements brodés traditionnels - même dans des scènes de travail - accompagnés de bijoux. Les coiffes se diversifient et le fichu noué dans le cou à la manière ouvrière n'est plus la seule possibilité [ill. 3]. Par ailleurs, cette évolution du tipazh remettant à l'honneur les costumes traditionnels correspond parfaitement un autre impératif de Tchaikov: la représentation presque ethnographique particulièrement visible dans les représentations

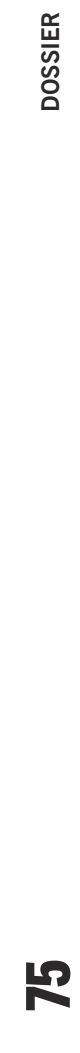

4. Tableaux de Natalia

au musée Russe :

5. Groupe sculpté pa

avant notre ère, en

l'honneur d'Harmodios

Aristogiton, les deux

la démocratie en

poignardant le tyran

Hipparque. Une copie

est conservée au musée

archéologique de Naples. 

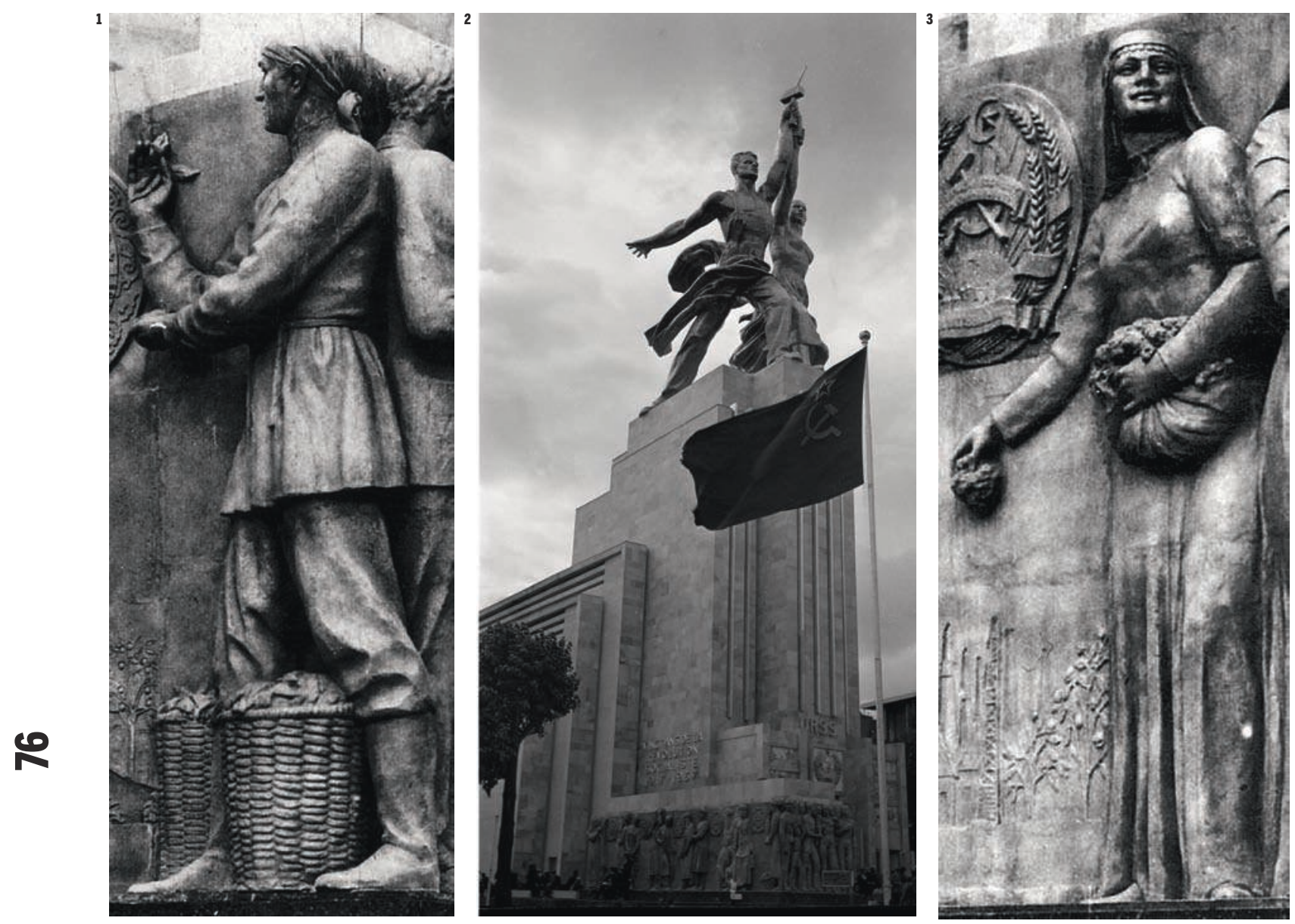

1. Joseph Tchaikov, Relief de la République socialiste soviétique de Géorgie, propylées du pavillon soviétique,1937.L'homme est représenté portant la tunique traditionnelle serrée à la taille par une corde et un pantalon rentré dans une paire de bottes. Tenant dans ses mains des feuilles de thé,

il remplit les deux paniers à ses pieds, renvoyant à une culture très répandue en Géorgie.

2.Vue du pavillon

soviétique : au premier plan, le propylée et les sculptures de Joseph Tchaikov.

3. Joseph Tchaikov, Relief de la République socialiste soviétique d'Azerbaïdjan, propylées du pavillon soviétique, 1937. Représentée en pleine

récolte du coton, la

femme azérie porte, sur son foulard, une coiffe ornée de petites médailles. Lesculpteur a même ajouté un collier orné d'une pierre et une paire de boucles d'oreille.

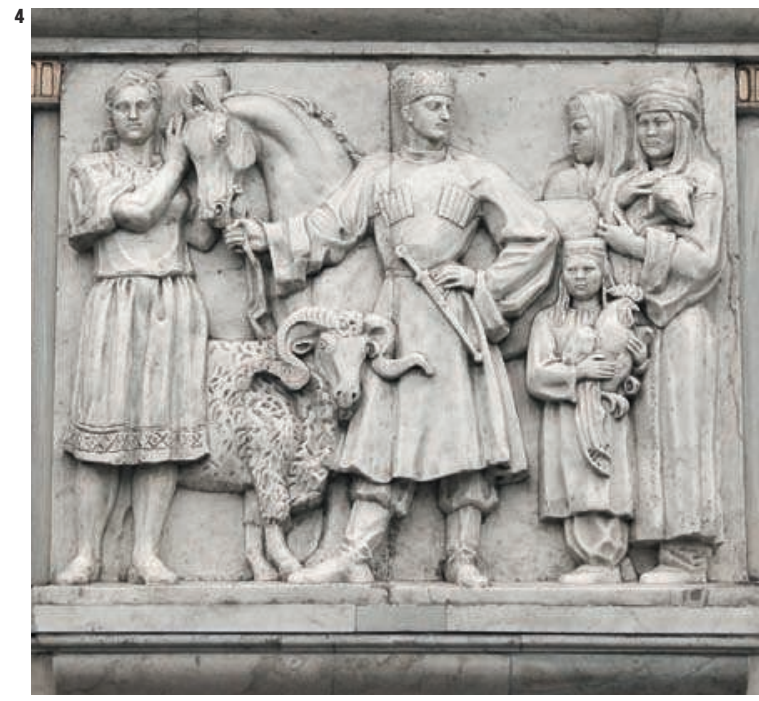

4. Georgi Motovilov,

L'Agriculture, Moscou

station de métro

"Elektrozavodskaia ",,

inaugurée en 1944.

5. Georgi Motovilov,

Reliefs pour l'entrée

de l'Exposition agricole

de 1939.

6. Joseph Tchaikov,

Le Passé (Paysan avec

son araire), musée Russe,

Saint-Pétersbourg.

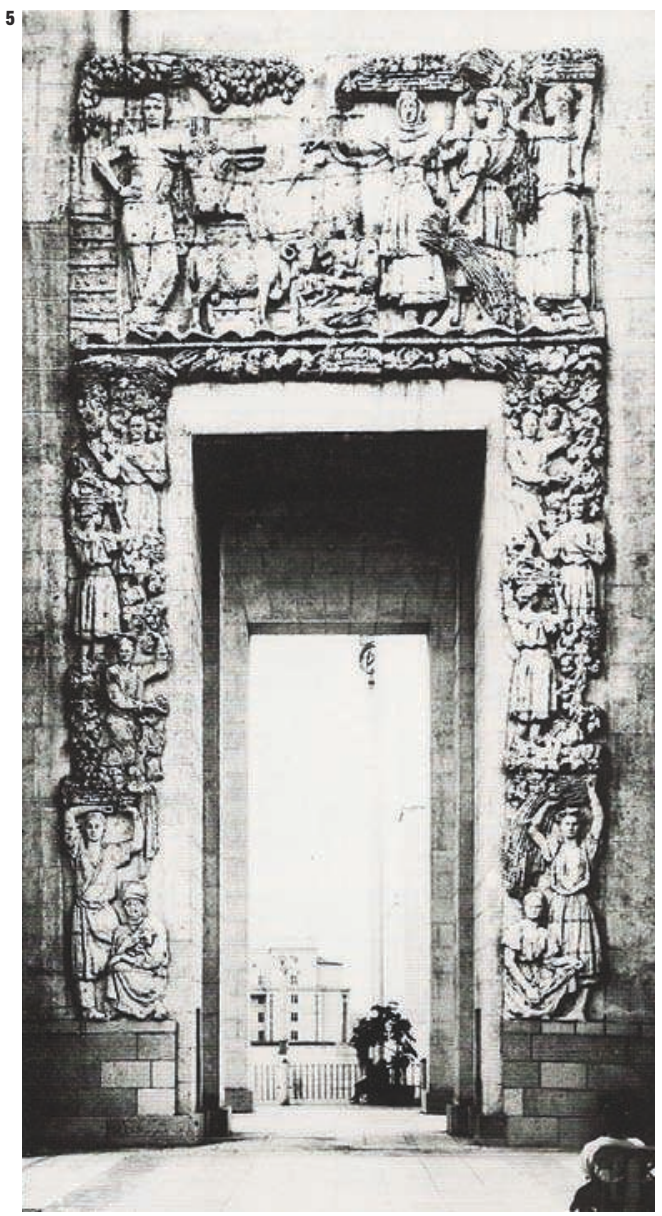


des personnages doit mettre en avant la richesse et l'étendue du territoire de l'URSS.

\section{Une allégorie du territoire}

Les paysans vont être la figure privilégiée pour montrer l'ampleur du territoire de l'URSS. Tout comme la diversité des peuples, la variété des types d'activité agricole est un moyen de souligner cette immensité. Tchaikov a fait le choix d'une grande lisibilité, exposant clairement armoiries officielles, personnages et ressources locales, le tout renforcé par la composition en frise, très systématique. Chaque visiteur de l'exposition devait être en mesure d'identifier les différentes républiques représentées.

Ces reliefs ont été réalisés à une période charnière, juste avant la fin de la politique d'ouverture aux nationalités constituant l'URSS. L'année 1937 correspond au début des grandes purges et à un assaut contre les nationalités, qui s'exprimera par une politique de russification intense. Les sculptures du pavillon soviétique ont donc bénéficié d'une ultime marge de liberté : l'unité recherchée n'excluait pas encore l'expression des identités culturelles locales. Au contraire, le monument devait affirmer l'union des peuples de l'URSS tout en valorisant ces identités culturelles. L'artiste devait à la fois rendre visible le caractère universel de l'homme soviétique, et montrer ostensiblement les différents types géographiques et sociaux qui le constituaient. Tentant de trouver un équilibre entre le local et l'universel, Tchaikov a apporté un soin presque ethnographique aux visages, aux costumes et aux attributs de chaque personnage. Cette allégorie du territoire à travers la représentation des différents peuples de l'URSS et la mise en valeur particulière des paysans se retrouve à la même époque chez d'autres artistes comme le sculpteur Georgi Motovilov (1892-1963) [ill. 4]. Réalisé dans le cadre d'un programme décoratif pour la station de métro «Elektrozavodskaia», le relief L'agriculture montre lui aussi des paysans aux activités et aux types variés, dans un style proche des reliefs antiques qui n'est pas sans rappeler le travail de Tchaikov?.

\section{La fiction de l'abondance}

Outre leur participation à une représentation allégorique du territoire, les paysannes des reliefs du pavillon soviétique peuvent également être considérées comme des symboles de la fertilité et de l'abondance de la mère patrie - rodina en russe, vient du verbe rodit', «mettre au monde ». Il s'agit de mettre en scène une abondance devenue l'un des objectifs du deuxième plan quinquennal. À l'achèvement du plan, sa célébration est omniprésente et fait les gros titres des journaux bien qu'elle soit totalement fictive. Gerbes de blés, ballots de coton et paniers de pommes rendent donc ici hommage aux réussites de la collectivisation de l'agriculture menée par Staline qui proclame en 1937: « La vie est devenue meilleure, la vie est devenue plus gaie ».
L'allégorie du territoire rejoint la fiction de l'abondance de façon encore plus visible deux ans plus tard, le $1^{\mathrm{er}}$ août 1939 , lors de l'ouverture de la grande Exposition agricole de l'URSS. Regroupant des pavillons pour chaque république - mais aussi un pavillon du maïs ou de la viande - elle doit montrer l'accomplissement de l'agriculture soviétique, à grands renforts de peintures monumentales, de groupes sculpturaux, de photographies et de diagrammes variés. Cette exposition prolonge et parachève les reliefs de Tchaikov. L'entrée principale de l'exposition - aujourd'hui la porte sud du centre panrusse des expositions $(\mathrm{BB} Ц)^{\mathbf{6}}$ - était décorée par des reliefs du sculpteur Georgi Motovilov. Ces sculptures, aujourd'hui disparues, sont les héritières de l'œuvre de Tchaikov [ill. 5]. Celui-ci livre d'ailleurs, à l'occasion de cette exposition, une sculpture en plâtre teinté : Le Passé (Paysan avec son araire) [ill. 6]. Un paysan au visage émacié tient d'une main lasse son araire. Le style presque expressionniste renforce la sensation du travail difficile. Pieds nus, harassé de fatigue, il incarne la période précédant les miracles de la mécanisation de l'agriculture.

Créer des rêveurs qui rêveraient de rêves socialistes, tel est l'objectif poursuivi par le réalisme socialiste selon l'historien de l'art Boris Groys (Groys et al., 2003). À travers ses représentations de paysans, Tchaikov nous propose bel et bien un rêve : celui d'un territoire immense et profondément uni, d'une abondance produite par la collectivisation de l'agriculture, d'un monde nouveau où la vie serait devenue meilleure et plus gaie, d'une œuvre d'art qui changerait l'humanité.
6. Le Centre panrusse des expositions accueille pavillon soviétique $\mathrm{d}$ 1937, abritant un musée dédié à l'art soviétique des années 1930.

7. Photographies des sculptures de Motovilov pour la station

«Elektozavodskaya », disponibles en ligne sur http://huck-d.livejournal. com/132677. html?thread $=1239365$.
Références bibliographiques

Bonnell V., 1997, Iconography of Power, Soviet Political Poster under Lenin and Stalin, Berkeley et Los Angeles, University of California Press.

Dufils A., Gentili F., VACHER M., 2011, « Les sculptures du pavillon soviétique de l'Exposition internationale de 1937 », Aden, Paul Nizan et les années 1930, 10, p. 207-233.

Gentili F., 2010, Baillet-en-France : glacière du Parc du Château. Statues soviétiques de l'exposition de 1937 Rapport final d'opération, Inrap, $229 \mathrm{p}$.

GENTILI F., 2011, «Des héros soviétiques dans la glacière», Historia, $\mathrm{N}^{\circ} 779$, p. 11

GENTILI F. VACHER M., à paraître, Une archéologie de l'art soviétique en France : les sculptures du pavillon soviétique de l'Exposition Internationale de 1937 ", in Mendelson J. (DIR.), Encounters with the 1930's, Catalogue d'exposition, Museo Nacional Centro de Arte Reina Sofía, Madrid.

Groys B., Hollein M. (DIR.), 2003, Dream Factory Communism, The visual culture of the Stalin Era, Catalogue d'exposition, Schirn Kunsthalle, Francfort

Le Monde paysan dans l'art russe, 2005, Catalogue d'exposition, Musée Russe, Saint-Pétersbourg, Palace Éditions.

VAudour C. (DIR.), 2011, Ruines et vestiges, Catalogue d'exposition, Musée archéologique départemental du Val d'Oise. 